\title{
Boxer Fracture: An Unusual Presentation
}

\author{
Obaid Al-Meshal", Doaa Andejani \\ Division of Plastic Surgery, King Abdulaziz Medical City \\ *Corresponding author: almishal_obaid@hotmail.com
}

Received June 13, 2015; Revised July 21, 2015; Accepted September 15, 2015

\begin{abstract}
We describe a very unusual presentation of the so called boxer fracture. We present a young patient who had a marked volar displacement, proximal migration and flipping of his subcapital metacarpal fracture of the little finger that was managed surgically. This case report illustrates this rather rarepresentation.
\end{abstract}

Keywords: metacarpal bones fracture, metacarpal bones surgery, boxer's fracture

Cite This Article: Obaid Al-Meshal, and Doaa Andejani, "Boxer Fracture: An Unusual Presentation.” Global Journal of Surgery, vol. 3, no. 2 (2015): 24-26. doi: 10.12691/js-3-2-2.

\section{Introduction}

Often associated with aggression, boxer fractures are fairly common. Due to the pre- existent volar angulation in the metacarpal head, the majority of these fractures present with varying degrees of volar angulation of the distal fragment. There is no clear consensus about the management of these fractures [1,2]. In this article we are presenting a very rare type of the boxer fractures in which open reduction and fixation was a must.

\section{Case Report}

A 17-year-old boy, who is otherwise in good health presented to our accident and emergency department. Patient was hit with a wooden bat during an altercation.

He complained of pain, swelling along the ulnar aspect of the right hand. Physical examination showed swelling, discoloration, normal neurovascular exam, and limited range of motion in the little finger (Figure 1). Appropriate analgesia was given and the patient was sent with an arm sling to the X-ray department.

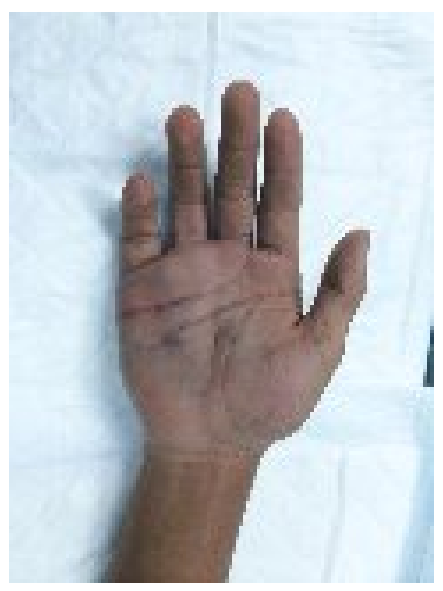

Figure 1.
X-ray showed subcapital metacarpal fracture of the right little finger with marked volar displacement, proximal migration and 180 degrees flipping (Figure 2Figure 3).

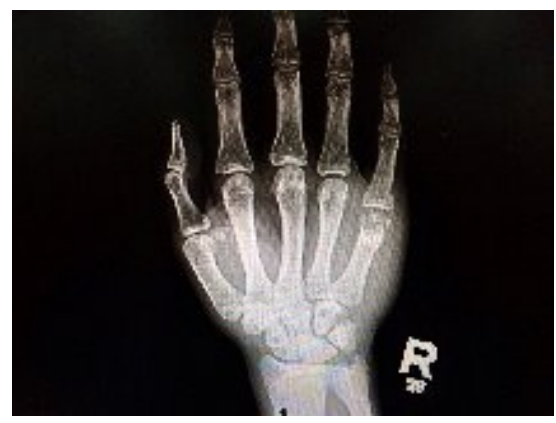

Figure 2.

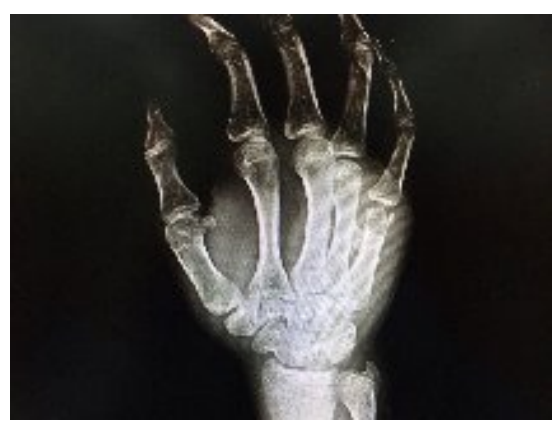

Figure 3.

Patient was admitted and planned for surgical intervention. Under general anaesthesia and with proper Tourniquet, volar Brunner incision was marked and the skin was incised. The fractured piece was found volar to the metacarpal shaft and trapped between the neurovascular bundle (ulnar) and the flexor tendon (radial) having lost its periosteal attachment to the metacarpal shaft (Figure 4). Reduction attempted, the distal fragment was replaced as a free bone graft and maintained in place with two percutaneous crossing $\mathrm{K}$ wires (Figure 5-Figure 6). Volar splint was applied. 


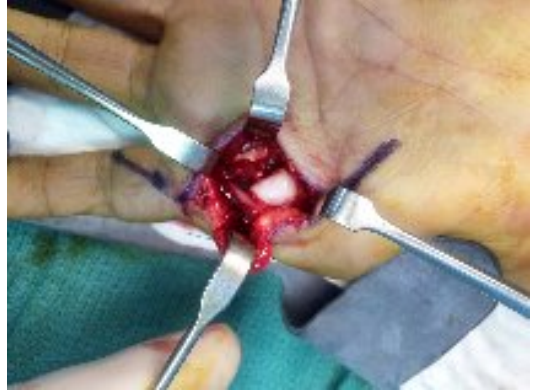

Figure 4.

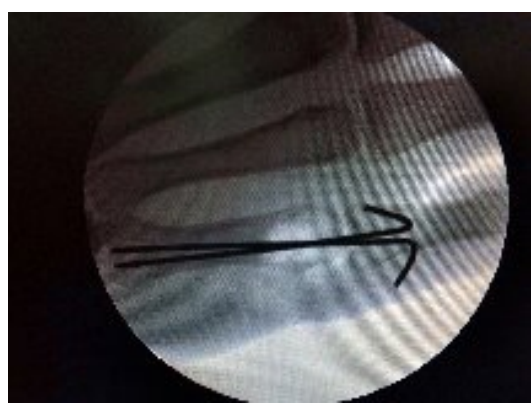

Figure 5 .

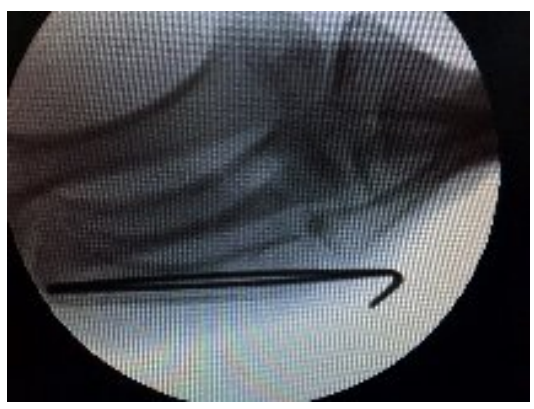

Figure 6 .

Patient had an uncomplicated post-operative course, attended regular follow ups and the $\mathrm{K}$ wires were removed in 4 weeks' time. He was sent to the hand physiotherapist and subsequent follow up showed decent return to preinjury hand function (Figure 7-Figure 8).

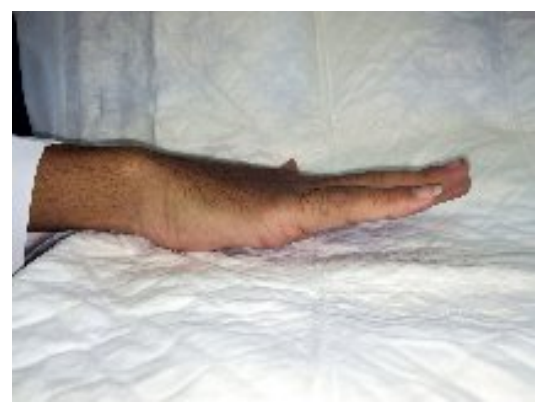

Figure 7.

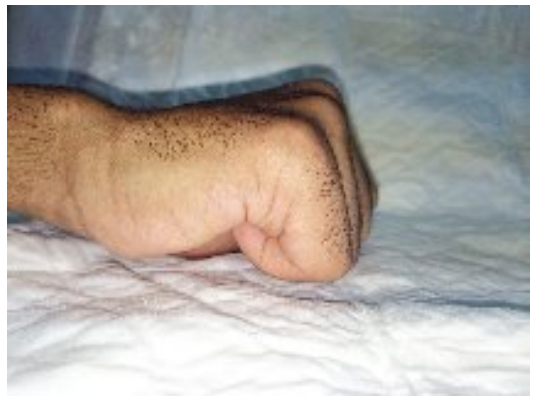

Figure 8.
Radiographic images at two months' time showed good alignment with no evidence of resorption nor avascular necrosis of the distal fragment (Figure 9-Figure 10).

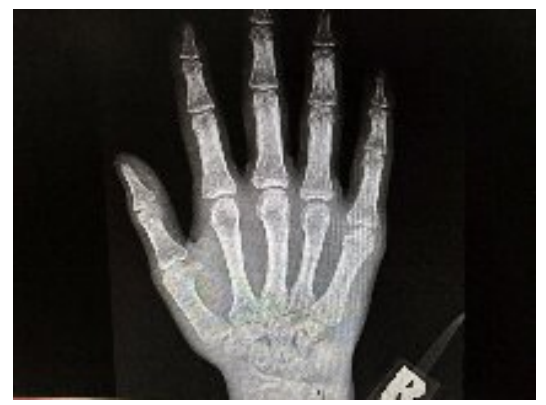

Figure 9.

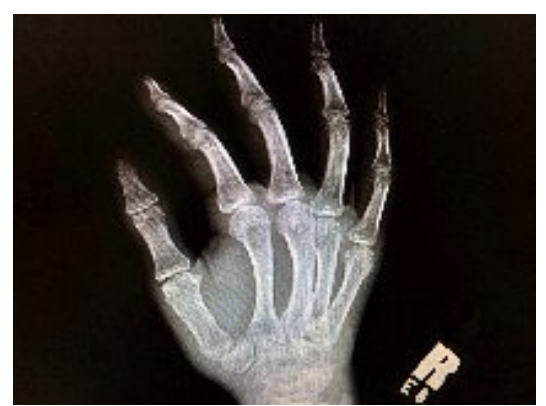

Figure 10.

\section{Discussion}

Metacarpal fractures account for $18 \%$ of all fractures of the hand and forearm [3]. The little finger is the most commonly involved digit with the subcapital fracture accounting for $25 \%$ of all metacarpal fractures [4]. It tends to occur in the young and active males and is often sustained through aggression. The little finger is notoriously prone to being fractured at the neck, usually as a result of an axial loading from a punching mechanism and hence the name "Boxers fracture"' was given to it despite the fact that professional boxers rarely get this type of fracture [1].

Metacarpal neck fractures usually occur when a clenched MP joint strikes a solid object resulting in dorsal angulation of the apex. Intrinsic muscles lie volar while crossing the MP joint and maintain the flexed metacarpal head posture [2].

The majority of the subcapital metacarpal little finger fractures are displaced with varying degrees of angulation. Our case presented with volar displacement, proximal migration of the fractured piece and 180 degrees flipping. Our literature review didn't yield any reported cases with such presentation.

The management of these fractures is still a matter of debate. The degree of palmar angulation deemed acceptable in the literature ranges between 20-70 degrees [2]. Some surgeons agree that considerable angulation of up to 70 degrees in these fractures doesn't seem to affect the hand function and it's an acceptable practice accordingly to attempt manipulation only if the angulation exceeds 40 degrees [5]. The Jahss manoeuvre remains the best technique in attempting closed reduction when angulation exceeds the acceptable limits, pseudoclawing or rotational deformities are evident [6]. There is still no 
agreement about maintaining the position of the boxer fracture post reduction. Methods vary from immobilisation in a plaster of Paris ulnar gutter to functional taping with full mobilisation to full dynamic treatment $[2,7,8,9]$. The General consensus is that surgical intervention is indicated in open fractures, instability and for multiple fractures. Methods of fixation vary between $\mathrm{K}$ wires, lag screws and plate fixation [10]. In our case, the distal fragment was completely detached from the metacarpal shaft's periosteum and we decided to replace it as a bone graft. Percutaneous $\mathrm{K}$ wires were chosen to minimize the chances of resorption of the distal fragment.

\section{Conclusion}

In cases with significant displacement of boxer fractures; surgical intervention with open reduction and fixation is needed specially if tendon entrapment is suspected. Healing of these types of fractures is good if managed well even if the distal fragment is attached as a free bone graft.

\section{References}

[1] Ring and little finger metacarpal fractures: mechanisms, locations, and radiographic parameters. Soong M, Got C, Katarincic J. 2010, J Hand Surg Am, pp.Aug;35(8):1256-9.

[2] Immediate mobilization gives good results in boxer's fractures with volar angulation up to 70 degrees: a prospective randomized trial comparing immediate mobilization with cast immobilization. Markwin G Statius Muller, Rudolf W poolman, M Julie Van
Hoogstraten, E philip Steller. 2003, Arch Orthop Trauma Surg, pp. 123:534-537.

[3] The frequency and epidemiology of hand and forearm fractures in the United States. Chung KC, Spilson SV.2001, J Hand Surg Am, pp. Sep;26(5):908-15.

[4] Antegrade inramedullary splinting or percutaneous retrograde crossed pinning for displaced neck fractures of the fifth metacarpal?. Schadel-Hopfner M, Wild M Windolf, Linhart W. 2007, Arch Orthop Trauma Surg, pp. 127 (6) 435-440.

[5] Fifth metacarpal fractures in a compensation clinic population. A report on one hundred and thirty-three cases. Hunter JM, Cowen NJ. 1970, J Bone Joint Surg Am, pp. Sep; 52(6): 1159-65.

[6] Charles S. Day, Peter J. Stern. fractures of the metacarpals and the phalanges. [book auth.] Robert N. Hotchkiss, William C. Pederson, Scott H. Kozin Scott W. Wolfe. Greens's operative hand surgery. s.l.: Churchill Livingstone, 2010, pp. 239-290.

[7] Immediate mobilization of fractures of the neck of the fifth metacarpal. M Arafa, J Haines, J Noble, D Carden. 1986, Injury, pp. 17:277-278.

[8] The treatment of fractures of the ring and little metacarpal necks, a prospective randmoized study of three different types of treatment. Hansen, P B Hansen and T B. 1998, Journal of Hnad Surgery, pp. 245-247.

[9] Three cast techniques for the treatment of extra-articular metacarpal fractures. Jeff Tavassoli, Robert T Ruland, Christopher J Hogan, David L Cannon. 2005, Journal of Bone and Joint Surgery, pp. 87: 2196-2201.

[10] The current practice of the managment of little finger metacarpal fractures, a review of the literature and results of a survey conducted among upper limb surgeons in the United Kingdom. A Sahu, S S Gujral, S Barta, S P Mills, M S Srinivasan. 2011, Hand Surgery, pp. 17:55-63.

[11] Tendon interposition in boxer's fracture, an unusual cause of nonunion and extensor dysfunction of the little finger. Johsy S, Ebrahim S, Iossifidis A. 2005, Injury Extra, Volume 36, Issue 7, pp. 237-238. 